\title{
Estudio metabólico en lactantes desnutridos alimentados con harina de garbanzos
}

DRES. : SERGIO JARPA $Y$ *, JORGE MARTNER $G . *$.

INTRODUCción. La rápida disminución de la lactancia materna y la insuficiente producción de leche de vaca en nuestro país, han hecho obligatoria la importanción de grandes cantidades de este producto que cada año se adquiere a un precio más elevado en el mercado intersacional.

Por acuerdos que datan de más de 15 años la alimentación del lactante chileno, atendido en su gran mayoría en los consultorios del Servicio Nacional de Salud, se efectúa con leche semidescremada (12\% de materia grasa) y en diferentes publicaciones (1) (2) (3) (4) se ha hecho notar lo inadecuado de esta medida, especialmente en los lactantes menores de 4 meses, por ser una ctapa de muy rápido crecimiento. Su uso, en las dosis habituales de $150 \mathrm{gr} / \mathrm{Kg} /$ día diluída al $10 \%$ y con $5 \%$ de sacarosa, constituye una alimentación "de alto costo, de baja eficiencia y distrofiante" (5).

De acuerdo con estos estudios diferentes inves. tigadores han ensayado un variado tipo de sustitutos de la leche de vaca para ser utilizados en la alimentación de pre-escolares (6) (7) (8) con lo cual la escasa cantidad de leche se reservaría íntegra para el lactante, podría administrarse con mayor contenido graso y se ahorrarian enormes sumas de dinero en su importación.

'También se han ideado fórmulas para el tratamiento del lactante con diarrea basado en el efecto astingente que tendrían algunos de sus elementos (9), o quizás en la menor cantidad de lactosa que tendría una de estas fórmulas (10) al reemplazar una apreciable cantidad de leche por otro alimento no lácteo.

Hace algún tiempo tuvimos oportunidad de analizar el efecto metabólico sobre el niño

\footnotetext{
- Serricio Nutrición y Laboratorio de Investigaciones, Hospital "Luis Calvo Mackenna".

** Departamento de Nutrición y Tecnologia de los Alimentos, Area Sur - Liviversidad de Chile.
}

de una de estas dietas y pudimos comprobar como, si bien mejoraba el aspecto de las deposiciones, aumentaba el volumen de ellas, se limitaba y dificultaba la absorción normal de proteínas y disminuía la ganancia de peso en forma siguificativa, pudiendo liegar a constituirse, cuando su uso era prolongado, en un alimento distrofiante (11).

Basado en estos estudios nos decidimos a investigar los cfectos metabólicos de la llamada $\mathrm{Ha}-$ rina de Garbanzos (G. L.) en un grupo de lactantes desnutridos hospitalizados en el Servicio de Nutrición del Hospital "Luis Calvo Mackenna" de Santiago de Chile, ya que su uso, de acuerdo con lat experiencia clínica únicamente, aparecía preconizado por las autoridades del Servicio Nacional de Salud.

Material Y MÉtodos. Se estudian 5 lactantes menores de 1 año (entre 5 y 7 meses de edad) con un grado de Desnutrición entre 43 y $74 \%$ del peso ideal y cuyos pesos varían desde 2.800 a 4.520 grs.

En todos ellos el motivo de ingreso al hospital fue Diarrea Aguda y Desnutrición.

Después de pasado el cuadro agudo se les sometió a la alimentación curativa en base a leche semidescremada adicionada de aceite, harina (maicena) y azúcar, que es de uso corriente en nuestro Hospital. Todos recibieron vitaminas A., C, y D. en cantidades adecuadas. Salvo diarrea moderada en 2 de ellos con cultivo y examen parasitológico seríado de deposiciones negativo, ninguno presentó infecciones ni tuvo otras enfermedades intercurrentes durante el periodo de estudio.

Con la alimentación indiçada, mantenida por un promedio de 26 días ( 22 a 33 ), se sometió a 4 de ellos a un primer período de balance de acuerdo a técnicas descritas (12) y luego se cambió bruscamente (todas las mamaderas el mismo 
día) a la alimentación de prueba (Harina de Garbanzos), para someter a todos los niños a un seguindo período de balance metabólico, de 5 días de duración, diez días después de su iniciación. Al términar este segundo período nuevamente se cambió, tambiên en forma brusca, a la alimentas. ción que recibían antes del G. L., cambios que se efectuaron para observar la tolerancia en cuanto a aceptabilidad de las dietas, calidad de las deposiciones y curva ponderal.

El tiempo transcurrido entre el primer y el segundo período de balance fue de un promedio de 13 días (10 a 15 días). El peso y la temperatura de los niños fue controlado diariamente.

Los análisis de las muestras de alimentos, deposiciones y orina, en lo concerniente a Nitrógeno, Grasas, Agua y Electrolitos, así como las de sangre, han sido previamente descritos (12).

El análisis químico efectuado al contenido de las mamaderats utilizadas en la alimentación de estos niños demuestra, por cada 100 gr. preparados, la constitución que se aprecia on la Tabla No $1 *$

TABLA No 1

ELEMENTOS CONTENIDOS EN LA DIETA (100 gr.

\begin{tabular}{|c|c|c|}
\hline & Leche & Gl. \\
\hline Proteínas: & 2,9 gr. \% & $3,2 \mathrm{gt} . \%$ \\
\hline Grasas: & $4,1 \mathrm{gx} . \%$ & 2,3 gr. $\%$ \\
\hline H. de C.: & 10,2 gr. $\%$ & 10,3 вг. $\%$ \\
\hline Agu: : & 82.8 gr. $\%$ & 842 gr. $\%$ \\
\hline Na.: & $20.1 \mathrm{mEq} / 1$ & $11,8 \mathrm{mEq} / 1$ \\
\hline K.: & $33,4 \mathrm{mEq} / 1$ & $44,7 \mathrm{mEq} / 1$ \\
\hline Cl.: & $25.8 \mathrm{mEq} / 1$ & $15,5 \mathrm{mEq} / 1$ \\
\hline
\end{tabular}

Resultados. Todos los niños recibieton las diferentes alimentaciones sin inconvenientes, no presentaron vómitos ni meteorismo abdominal y las deposiciones normales en 3 de ellos no se alteraron con la nueva fórmula alimenticia, en tanto que 2 lactantes que presentaban una diarrea moderada antes de iniciar cl cstudio no presentaron mejoría de las deposiciones con los cambios dietéticos efectuados.

En la tabla $\mathrm{N}^{\circ} 2$ se establece una comparación entre el volumen de las deposiciones que tuvieron estos niños mientras se alimentaron con leche y posteriormente con GL. (se agrega el volumen ingerido en cada período) expresado on $\mathrm{gr} / \mathrm{Kg} /$ día.

\footnotetext{
* N. de la R. Se hace notar que olros autores nacionales (10) han usada formulas a base de liarioa de garbanzos y lache (G. L.) adicionadas de DL metionjana yna mexcla de vitaminas y minerales, clementos quc no contenía el G. L. ulilizado por el SNS para el tratamiento de la diarrea.
}

T A B L A Nơ 2

VOLUMEN DE LAS DEPOSICIONES CON 2 DIETAS DIFERENTES (SE AGREGA VOLUMEN DE LO INGERIDO; GR/KG/DIA

\begin{tabular}{lcrrr}
\hline & $\begin{array}{c}\text { Leche } \\
\text { Ingestion }\end{array}$ & Deposición & $\begin{array}{c}G \text { I. } \\
\text { Ingestión }\end{array}$ & Deposición \\
\hline P. H. & - & - & 153,2 & $\mathbf{5 7 , 0}$ \\
C. L. & 154,3 & 35.2 & 157,2 & $\mathbf{4 9 , 5}$ \\
H. C. & 132,1 & 9,5 & 141,9 & $\mathbf{3 7 , 3}$ \\
F. M. & 138,3 & 11,9 & 143,4 & $\mathbf{4 1 , 4}$ \\
A. V. & 154.1 & 4,6 & 142,5 & $\mathbf{2 4 , 6}$ \\
\hline
\end{tabular}

En valores promedios la Ingestión fue similar ya que, mientras se alimentaron con leche recibieron 144,7 gr. y con GL. 147,6 gr.

El niño P. $H$. no tuvo un Balance Metabólico de control. El niño $C$. L. presentaba diarrea duränte el balance con leche $(35,2$ gr. de deposiciones $/ \mathrm{Kg} /$ día) la que aumentó a $49,5 \mathrm{gr} / \mathrm{Kg} /$ día con la alimentación GL. Los restantes niños $(H$. C. - F. M. - A. V.) que presentaban deposiciones normales con la leche (promedio $8,6 \mathrm{gr} / \mathrm{Kg} /$ día) atumentaron 4 veces el volumen de las deposiciones (34,4 gr.) al ingerir GL., si bien las características físicas (forma, consistencia, color, olor, etc.) vrait normales.

Las variaciones en la curva de peso se exponen en la tabla siguiente.

$$
\text { T A B L A No } 3
$$

VARIACION DE LA CURVA PONDERAL DURANTE EL PERIODO DE ESTUDIO. GRAMOS EN 10 DIAS (PERIODOS CONSECUTIVOS)

\begin{tabular}{lccc}
\hline Niño & $\begin{array}{c}\text { Leche } \\
\text { dias antes } \\
G L .\end{array}$ & $G L$. & $\begin{array}{c}\text { Leche } \\
\text { 10 dias des } \\
\text { pués } G L .\end{array}$ \\
\hline PH. & -100 & 0 & \\
C. L. & +130 & -80 & \\
H. C. & +120 & -40 & +320 \\
F. M. & +170 & +110 & +220 \\
A. Y. & +270 & +170 & \\
\hline
\end{tabular}

Como se puede deducir de lo expuesto en la tabla $N^{\circ}$ 3, en los 10 días inmediatamente anteriores a la alimentación con GL., estando alimentados con leche, el promedio de ganancia de peso de los 5 niños fue de 11,8 gr. diarios, cifra quc es casi 3 veces superior a la obtenida en los 10 dias siguientes, mientras recibieron la alimentación GL. (3,2 gr. diarios). 
En la tabla Nơ 4 se pueden observar las variaciones en el consumo de liquidos ingeridos durante el tiempo que estuvieron los lactantes sometidos a las diferentes dietrs que se analizan.

TA B L A Nọ 4

VOLUMEN DE AGUA CORREGIDA PARA SOL.IDOS (AGUA COMO TAL Y DE LA ALIMENTACION) $M L / K G / D I A$

\begin{tabular}{|c|c|c|c|c|c|c|}
\hline Niño & $\begin{array}{c}\text { Inges } \\
\text { tibn }\end{array}$ & $\begin{array}{l}\text { Leche } \\
\text { Orina }\end{array}$ & $\mathrm{mOsm} / 1$ & $\begin{array}{c}\text { Itgess } \\
\text { tiont }\end{array}$ & $\begin{array}{c}G L . \\
\text { Orina }\end{array}$ & $\mathrm{mosm} / \mathrm{s}$ \\
\hline P.H. & & & & 195,0 & 84,6 & (393) \\
\hline C.L. & 149.1 & 54,7 & $(600)$ & 174,4 & 41,4 & (650) \\
\hline H.C. & 131,5 & 55,4 & (428) & 166,4 & 51,2 & (493) \\
\hline F.M. & 135,3 & 66,6 & (385) & 158,4 & 65,1 & (615) \\
\hline A.Y. & 160,5 & 101,0 & (319) & 161,9 & 82,0 & (474) \\
\hline
\end{tabular}

Al relacionar la cantidad de agua ingerida (agua de la alimentación, corregida para sólidos, y agua como tal) con el volumen de orina, se observa que este volumen disminuye levemente con GL. de un promedio de $69,4 \mathrm{ml} / \mathrm{Kg} /$ día a $64,9 \mathrm{ml} /$ $\mathrm{Kg} /$ día. Por otro lado aumenta la ingestión de agua a tomat como tal mientras se alimenta con GL., llegando a un promedio total de $172,2 \mathrm{ml} /$ $\mathrm{Kg} /$ día, en tanto que el de la alimentación control alcanza sólo a $144.1 \mathrm{ml} / \mathrm{Kg} /$ día.

Se observa también que la osmolaridad de la orina aumenta mientras recibe la alimentación GL., incluso en el niño C. L. que presentaba diarrea en el período de control.

En lo referente al metabolismo Nitrogenado, en la tabla $N$ : 5 se observan 2 aspectos importantes que destacan nítidamente el primero de ellos es la mayor cantidad de nitrógeno ingerida mientras estuvieron sometidos a la dieta GL. y el scgundo es la diferencia en el porcentaje de absor-

TABLA No 5

METABOLISMO NITROGENADO ( $\mathrm{mgr} / \mathrm{Kg} / \mathrm{dia}$ )

\begin{tabular}{lcccccc} 
Niño & $\begin{array}{c}\text { Inges- } \\
\text { tión }\end{array}$ & $\begin{array}{c}\text { Leche } \\
\text { Absorción }\end{array}$ & \% Abs. & $\begin{array}{c}\text { Inges- } \\
\text { tión }\end{array}$ & $\begin{array}{c}G L . \\
A b s .\end{array}$ & $\%$ Abs. \\
\hline P.H. & - & - & - & 766 & 495 & 64 \\
C.L. & 586 & 466 & 80 & 822 & 604 & 74 \\
H.C. & 581 & 518 & 89 & 753 & 596 & 79 \\
F.M. & 675 & 605 & 90 & 758 & 583 & 77 \\
A.V. & 690 & 634 & 92 & 775 & 540 & 70 \\
\hline
\end{tabular}

ción entre ambas alimentaciones, siendo significativamente inferior con GL.

Si bien el númcro de niños es pequeño, al promediar las cifras anotadas encontramos que con GL. ingieren una mayor cantidad de 125 mgr. de Nitrógeno/Kg/día en comparación con la dieta control y al mismo tiempo el porcentaje de absoción desciende desde un $88 \%$ con leche a un $75 \%$ con GL.

Balance Graso: Con ambas alimentaciones el porcentaje de absorción fue excelente, alcanzando hasta un promedio de $95 \%$ con GL.

Hay que destacar en este sentido el hecho de que, a pesar del aumento de volumen de las deposiciones, la pérdida de grasas en estos niños sea $\tan$ pequeña.

En el estudio del Balance de Sodio se hace evidente que estos lactantes, al ser alimentados con GL. tienen una menor ingestión, mayor pérdida por las deposiciones menor eliminación por la orina de este elemento, y menor retención en contraposición a cuando reciben la dieta de leche (Tabla Nọ 6).

TA B L A No 6

VALORES DE NA. INGERIDO Y ELIMINADO CON 2 DIETAS DIFERENTES ( $m E q / \mathrm{Kg} / \mathrm{dia})$

\begin{tabular}{|c|c|c|c|c|c|c|c|c|}
\hline \multirow[b]{2}{*}{ Nontse } & \multicolumn{4}{|c|}{ Leche } & \multicolumn{4}{|c|}{$G L}$. \\
\hline & Ins & Dcp. & Oriata & Ret. & Ing. & Dcp. & Orina & Ret. \\
\hline P.H. & - & - & - & 一 & 1,1 & 1,4 & 0,3 & $-0,6$ \\
\hline C.L. & 2,8 & 1.5 & 0,4 & $\because 0,9$ & 1,2 & 0,9 & 0.2 & +0 \\
\hline H.C. & 2,3 & 0.2 & 0,8 & $\therefore \quad 1,5$ & 1,9 & 0,8 & 0,2 & $+0,9$ \\
\hline F.M. & $2, n$ & 0,4 & 1,3 & $\therefore 1, \mathrm{~L}$ & 2,0 & 1,3 & 0.2 & $+0,6$ \\
\hline A.V. & 3,1 & 0,1 & 1,8 & $+1,2$ & 1,9 & 0,3 & 0,8 & +0. \\
\hline
\end{tabular}

La conjugación de una menor ingestión y una mayor pérdida por las deposiciones al ser alimentados con GL. es, indudablemente, el factor condicionante de la menor eliminación por la orina al recibir esta alimentación. Las concentraciones de sodio en la orina se mantienen muy bajas llegando uno de los niños (A. V.) a tener una concentración de $0,72 \mathrm{mEq} / 1$.

Balance del Potasio: En la tabla No 7 se destaca la mucho mayor ingestión de este elemento y la mayor pérdida por las deposiciones, al ser alimentados con GL. La retención es variable, siendo negativa en 2 niños y muy inferior a la que se obtiene con leche, especialmente si consideramos la cantidad ingerida.

Los valores sangúneos de los diferentes elementos analizados $(\mathrm{Na}-\mathrm{K}-\mathrm{Cl}-$ Uremia - Protei- 
TA B L A № 7

INGESTION Y ELIMINACION DE POTASIO CON 2 DIET AS DIFERENTES ( $\mathrm{mEq} / \mathrm{Kg} /$ dia)

\begin{tabular}{|c|c|c|c|c|c|c|c|c|}
\hline \multirow[b]{2}{*}{ Nombre } & \multicolumn{4}{|c|}{ Leche } & \multicolumn{4}{|c|}{$G L}$. \\
\hline & Ing. & Dep. & Orina & Ret. & Ing. & Dep. & Orina & Ret. \\
\hline P.H. & - & - & - & - & 6.6 & 4,7 & 2,1 & $-0,2$ \\
\hline C.L. & 4,6 & 2,4 & 1,0 & + 1,2 & 6,5 & 3,8 & 0,9 & $+1,8$ \\
\hline $\mathrm{H} . \mathrm{C}$. & 4,2 & 0.4 & 2,6 & $-1 \quad 1,2$ & 5,8 & 2,5 & 2,3 & $+1,0$ \\
\hline F.M. & 4,7 & 0.4 & 3,0 & $+1,3$ & 7.2 & 1,0 & 4,3 & $+1,9$ \\
\hline A.V. & 4,9 & 0,8 & 3,6 & . $\quad 0,5$ & 7,0 & 2,2 & 4,9 & $-0,1$ \\
\hline
\end{tabular}

nemia fueron normales y no revelaron alteraciones durante los períodos de estudio a que fueron sometidos estos lactantes.

Discusión. A pesar del escaso número de ninós estudiados se pueden sacar diferentes conclusiones de valor, por haber hechos que se presentan en forma constante e invariable en los diferentes pecíodos de estudio de todos ellos.

Lo que resalta más claramente es el gran aumento del volumen de las deposiciones mientras reciben la alimentación GL., y llama la atención que éste no se acompaña de cambios en otras características físicas de ellas, apreciables a simple vista.

Otro hecho que se hace evidente es Ja disminución del porcentaje de absorción de Nitrógeno cuando se alimentan con GL. Esto, por sí solo, no constituye un factor decisivo en la retención de este elemento cuando se le administra en las cantidades prescritas en este trabajo, porque, si bien el porcentaje de absorción es menor, la cantidad absoluta absorbida es semejante con ambas dietas, debido a la mucho mayor ingestión con GL. Si por cualquier motivo el niño jngiere una cantidad inferior de este alimento, este factor sería significativo ya que la cantidad absorbida sería insuficiente para las necesidades del organismo.

El uso de la Harina de Garbanzos ba sido preconizado para el tratamiento de la diarrea prolongada y como alimento curativo en la diarrea aguda del lactante. Pensamos que esta mezcla de un $20 \%$ de leche y un $80 \%$ de haritia de garbanzos dismiluye en forma apreciable la cantidad de lactosa que recibe el lactante, disminuyendo también la intolerancia a este disacátido descrita en niños con diarrea aguda o prolongada (13). La curvia de peso de algunos lactantes con diarrea aguda tratados con GL. en el Hospital Calvo Mackenna parece ser satisfactoria y en todo caso se observaría una rápida mejoría de la diarrea (14). Este hecho bien puede atribuirse a la suspensión total de la alimentación en las primeras horas, seguida de realimentación progresiva, pobre en lactosa, con agregado de abundante cantidad de agua y algunos electrolitos. La mantención de esta alimentación en lactantes desnutridos sin diartea, por un período de 10 o más días, se acompaña de una curva de peso insuficiente en comparación a la quc se obtiene con la alimentación clásica de leche y agregados de aceite más hidratos de carbono, que se utiliza en la reparación de la desnutrición.

Otro hecho que destaca en el análisis de los resultados obtenidos en estos niños es lo que se refiere al metabolismo del $\mathrm{Na}$. El GL. contienc una cantidad de este elemento muy inferior a la leche, lo sial, sumado a la mayor pérdida por las deposiciones determina una mínima eliminación por parte del riñón. Las cantidades llegan a ser tan pequeñas que en uno de los niños alcanzó una concentración menor de $1 \mathrm{mEq} / 1$. de orina. Este resultado, es obviamente, una reacción compensadora por parte de este órgano.

Para el balance del agua, el aumento de volumer de las deposiciones significa una mayor pérdida de este elemento no controlable por el organismo. La respuesta inmediata del riñón es la disminución del yolumen urinario, lo cual, a su vez, significa una menor capacidad para eliminar so. lutos, los cuales deben hacerlo en forma mucho más concentrada. En los niños de esta investigación este fenómeno fue oscurecido por el hecho de haber ingerido una apreciable mayor cantidad de agua.

La osmolaridad de la orina sube, aunque sin llegar a niveles muy altos, mientras reciben la alimentación de prueba y es un índice de la mayor sobrecarga renal a que están sometidos, a pesar de la mayor ingestión de líquidos.

Si la disponibilidad de agua para la función renal disminuye debido al aumento prolongado de pérdidas jor las deposiciones, se disminuye proporcionalmente la capacidad de reserva de este órgano ante pérdidas agudas de este elemento, como sucede en la diarrea, en el aumento de la pérdida insensible por fiebre o calor, en el ayuno, etc.

La mayor cantidad de potasio contenido en la dieta control es de esperar dada su composición principalmente vegetal. El comportamiento que de él hace el organismo hace que se pierdan grandes cantidades por orina y deposiciones llegando incluso a un balance negativo en 2 de estos niños, el segundo de los cuales no tenía diarrea durante el período de balance, to cual confiere mayor inportancia a este hecho.

Debido al corto tiempo que tuvo cada período de estudio no esperamos encontrar alteraciones en los valores sanguineos analizados, ya que su alteración, sin mediar enfermedades agudas intercurrentes, no cabría observarse en tan breve tiempo.

Del anćlisis del conjunto de los datos se puede deducir la existencia de una alteración en los balances de los elementos nutritivos, minerales y 
agua, que colocan al niño alimentado con GL. en una situación muy precaria frente a cualquier situación de emergencia como es una enfermedad, ya que puede hacer graves desequilibrios del metabolismo en situaciones que normalmente sería capaz de compensar.

Por la variedad de la alimentación a que están sometidos los pre-escolares y con mayor razón los escolares, el uso de esta mezcla dietética podría servii en ellos en reemplazo parcial y aún total de la leche de vaca, sin los inconvenientes que, como único alimento, provoca en el Jactante.

\section{REsUMeN}

1.- Mediante técnicas de balance metabólico se comparan 2 tipos de alimentación (Leche de vaca y Harina de Garbanzos) en 5 lactantes desnutridos.

2.- El volumen de las deposiciones es francamente superior al recibir la alimentación de prueba (GL).

3.- El progreso ponderal es deficiente mientras reciben esta dieta.

4.- En comparación con el perioda de control ingieren mayor cantidad de agua en tanto que los wolumenes urinarios son semejantes.

5.- Se aprecia una disminución del porcentaje de absorción de Nitrógeno, pese a una mayor ingestión, mientras reciben $G L$.

6.- El balance de $\mathrm{Na}$ revela una menor ingestión y menor retención, en comparación con la dieta control.

7.- Con la dieta en estudio ingieren mayor cantidad de $\boldsymbol{K}$ y la retención es menor.

\section{BiBLIOgRafía}

1.-Aiejandro Maccioni S. "Requerimientos Nutritivos. Rev. Chilena de Pediatría. XLIII: 25, 1972.

2.-Francisco Larrain B. "Requerimento de Grasa en el Lactante". Rev. Chilena de Pediatría. XLIII: 45, 1972.
3.-Holmun, R. T. Caster, W. O. and Wiese, H. F. "The essential fatty acid requirement of infants and the assesment of their dietary intake of linoleate by serum fatty acid analysis. Am. J. Clinical Nutr.: 14: $70,1964$.

4.-Contittes on Nutrition. Acad. Pediat. Citado por 5.

5.-Alejandic Maccioni, Ives Lacassie y Alfonso Salinas. "Fórmula láctea adaptada a los requerimientos calóricos y proteicos del lactante menor. Rev. Chilena de Pediatría. XLIII: 7, 1972.

6.--Sergio Jarpa Y., y Jorge Martner G. "Estudios Metabólicos en Lactantes Desnutridos alimentados con Harina de Pescado". Rev. Chilena de Pediatría. XL: $780,1969$.

7.-Jorgc Martner G., Sergio Jarpa Y. y Fernando Mönckeberg B. "Estudio Metabólico del Desnutrido alimentado con una nueva dieta de bajo contenido en Leche". Rev. Chilena de Pediatría. 44; 401, 1973.

8.- Enrique Yúnez, D. Ballester, C. O. Chichester and Ferrtando Mönckeberg B. A protein-rich mixture based on dry skim milk, toasted wheat flour, fish flour and sunflower meal". Proceeding Western Hemisphere Nutrition Congress III. 1971, pág. 377.

9.- Une nouvelle médication untidiarrheique, ta farine de caroube. Annales Pediatrici. 166: 113, 1946.

10.-E. Vallejos, P., M. E. Radrigún K., Ita Barja, Julia Araya, O. Solimano, M. A. Tagle y Patricia Muñoz. "Mamadera de Garbanzo (citer aretinium) cn el tratamiento de la diarrea prolongada del lactante desnutrido de tercer grado". Rev. Chilena de Pediatría. XLIII: 17, 1972.

11.- Jorge Martner G., Sergio Jarpa Y., Francisco Biancani $M$. "Estudio Comparativo entre una Leche Semidescremada y otra similar con agregado de Harina de Algarroba". Rev. Chilena de Pediatría 44, 407, 1973.

12.- Sergio Jarpa $Y$, y Jorge Martner G. "Organización de ura Unidad Metabólica". Rev. Chilena de Pediatría. XXXIII: 7, 1962.

13.-David M. Paige, Eduardo Leonardo, Angel Cordano, Julio Nakashima, Blanca Adrianzen and George Graham. "Lactose Intolerance in Peruvian Children: Effect of age and early nutrition". Am. J. Clin. Nutr. 25: 297, 1972.

14.- Abdalia Harún M. Hospital "Luis Calvo Mackenna'. Comunicación personal. 